\title{
Article \\ Argyrophilic Nucleolar Organizer Regions as New Biomarkers in ST-Elevation Myocardial Infarction
}

\author{
İbrahim Halil Damar ${ }^{1, *(1)}$ and Recep Eroz ${ }^{2}$ \\ 1 Department of Cardiology, Medical Faculty, Duzce University, Duzce 81820, Turkey \\ 2 Department of Medical Genetics, Medical Faculty, Aksaray University, Aksaray 68100, Turkey; \\ receperoz@aksaray.edu.tr \\ * Correspondence: ihdamar1@gmail.com; Tel.: +90-380-5421-390
}

check for updates

Citation: Damar, İ.H.; Eroz, R. Argyrophilic Nucleolar Organizer Regions as New Biomarkers in ST-Elevation Myocardial Infarction. J. Cardiovasc. Dev. Dis. 2022, 9, 58. https://doi.org/10.3390/jcdd9020058 Academic Editor: Maurice Van den Hoff

Received: 15 January 2022 Accepted: 11 February 2022 Published: 14 February 2022

Publisher's Note: MDPI stays neutral with regard to jurisdictional claims in published maps and institutional affiliations.

Copyright: (C) 2022 by the authors. Licensee MDPI, Basel, Switzerland. This article is an open access article distributed under the terms and conditions of the Creative Commons Attribution (CC BY) license (https:// creativecommons.org/licenses/by/ $4.0 /)$.

\begin{abstract}
Background: ST-elevation myocardial infarction (STEMI) is an inflammatory disease in which neutrophils, macrophages, and lymphocytes accumulate in the ischemic myocardium and have important functions. Nucleolar-organizing regions (NORs) are the site of the ribosomal genes composed of ribosomal DNA and proteins. We aimed to evaluate AgNOR proteins, which have never been studied in patients with STEMI in the literature. (2) Methods: A total of 140 participants (75 with STEMI and 65 volunteers without any diagnosis of acute coronary syndrome) were included in this study. Echocardiography was carried out, and mean AgNOR number and total AgNOR area/total nuclear area (TAA/TNA) were evaluated for all individuals. (3) Results: The mean AgNOR number and TAA/TNA ratio were significantly higher in the STEMI group than the control $(p<0.001)$. Statistically significant relations between both TAA/TNA ratio and mean AgNOR number and interventricular septal thickness, fasting blood sugar, creatinine, HDL, hemoglobin (g/dL), $\mathrm{WBC}(\mu \mathrm{L} / \mathrm{mL})$, monocytes, neutrophils, and neutrophil/lymphocyte ratio were detected $(p<0.05)$. Moreover, a statistically significant relation between LDL (mg/dL) and mean AgNOR number ( $p=0.005)$ was detected. (4) Conclusion: Both AgNOR protein amounts increase depending on the hypoxia that occurs in STEMI. The AgNOR proteins may thus be promising markers in STEMI.
\end{abstract}

Keywords: AgNOR; hypoxia; ischemia; STEMI

\section{Introduction}

Acute myocardial infarction is an inflammatory disease that usually develops on the basis of coronary artery disease [1]. Immune system cells, which infiltrate into the plaque from the early stages of atherosclerosis, increase inflammation by synthesizing and stimulating the molecules that accelerate the progression of the lesion [2]. During the acute myocardial infarction process, within the first five hours after reperfusion, neutrophils, as well as macrophages and lymphocytes, accumulate in the ischemic myocardium [3]. $\mathrm{T}$ lymphocytes have a key role in the pathophysiology of acute myocardial infarction (MI) within the cells of the immune system. T cells activate B lymphocytes, monocytes, macrophages, endothelial cells, and smooth muscle cells by releasing many cytokines, such as interferon-gamma $[4,5]$.

Nucleolar-organizing regions (NORs) are the site of the ribosomal genes composed of ribosomal DNA (rDNA) and proteins; some of them have argyrophilic features. After silver staining, NORs can especially be localized as black spots along the nucleolar space and are termed "AgNOR". Different studies on the importance of AgNOR proteins were performed in various cells such as hair root cells [6], buccal epithelial cells [7], lung cells [8], myocytes [9,10], muscle cells [11], etc. In those studies, the levels of AgNOR protein increased depending on the hypoxic condition caused by $\mathrm{CO}$ exposure and may have had protective effects against hypoxic conditions $[9,10]$.

In MI, myocardial ischemia/hypoxia occurs depending on acute coronary thrombosis. To the best of our knowledge, there are no studies evaluating AgNOR proteins in 
patients with MI. Thus, in the present study, we aimed to compare the AgNOR values of patients with ST-elevation myocardial infarction (STEMI) with those of people without acute coronary syndrome.

\section{Materials and Methods}

\subsection{Study Design}

A total of 140 participants who presented to the outpatient cardiology clinic of our institution were included in the study. The STEMI group consisted of 75 patients with a diagnosis of STEMI who underwent percutaneous coronary intervention, and the control group consisted of 65 volunteers without any diagnosis of acute coronary syndrome. The study protocol was approved by the local ethics committee (ethical approval code: 2021/62). Written informed consent was obtained from the participants. Uncontrolled hypertension, congenital heart disease, atrial fibrillation, severe valvular heart disease, hypothyroidism, hyperthyroidism, malignancy, and infection were determined as exclusion criteria. The diagnosis of STEMI was made under the guidance of the Fourth Universal Definition of Myocardial Infarction [12]. In patients who underwent percutaneous coronary intervention, primarily, the procedure only intervened in the responsible total lesion. Coronary artery stenosis was determined if the plaques caused $50 \%$ or more obstruction in the coronary lumen, while hemodynamically insignificant stenosis was determined if the lesions caused less than $50 \%$ stenosis. Blood was obtained from patients included in the study with a diagnosis of STEMI within the first six hours after the onset of chest pain. Diabetes mellitus was defined by use of antidiabetic therapy or fasting plasma glucose levels of $>6.94 \mathrm{mmol} / \mathrm{L}$ (>125 mg/dL). Hypertension was defined as antihypertensive drug use or blood pressure of $\geq 140 / 90 \mathrm{mmHg}$. Hyperlipidemia was defined as serum low-density lipoprotein level of $\geq 2.6 \mathrm{mmol} / \mathrm{L}$, triglycerides of $\geq 1.7 \mathrm{mmol} / \mathrm{L}$, total cholesterol of $\geq 5.2 \mathrm{mmol} / \mathrm{L}$, or the use of cholesterol-lowering drugs (1). Smokers were defined as people who continue to smoke currently. Laboratory findings (creatinine, low-density lipoprotein (LDL), high-density lipoprotein (HDL), triglyceride, total cholesterol level, and complete blood cell count (CBC)) and demographic features of the participants were recorded.

\subsection{Electrocardiography and Echocardiography}

Resting 12-lead ECGs of all patients were recorded using the NIHON KOHDEN Cardiofax ECG 1250K model (filter range, 0.05-150 Hz; AC filter, $60 \mathrm{~Hz}, 25 \mathrm{~mm} / \mathrm{s} 10 \mathrm{~mm} / \mathrm{mv}$ ). Echocardiography of the patients was performed using a Siemens Acuson SC2000 device. Cardiac anatomy, segmental wall motion abnormality, ejection fraction, and valve function were evaluated in accordance with the recommendations of the American Echocardiography Association with standardized projections [13].

\subsection{Coronary Angiography}

Selective right and left coronary angiography and PCI procedures were performed on patients in the STEMI group using the standard Judkin technique with a General Electric INNOVA 2100 IQ device. Coronary arteries were visualized in right and left oblique positions with cranial and caudal angulations.

\subsection{AgNOR Staining}

Blood samples of both the control and STEMI groups were taken and spread on clean slides. The slides were air dried for $15 \mathrm{~min}$ and fixed in absolute methanol for $5 \mathrm{~min}$ at room temperature. After that, the silver staining method with slight modification of the Benn and Perle protocol [14] and Lindner's technique [15] was performed for each slide. The solution, made by mixing one volume of $2 \%$ gelatin in $1 \%$ aqueous formic acid and two volumes of $50 \%$ silver nitrate, was dropped on the slides and incubated at $37^{\circ} \mathrm{C}$ for $15 \mathrm{~min}$ in the dark. After the incubation, the slides were rinsed with double distilled water. 


\subsection{Image Analysis of Mean AgNOR Number and Total AgNOR Area/Total Nuclear Area (TAA/TNA) Ratio}

Fifty nuclei for each slide were evaluated. Firstly, silver-stained lymphocyte cells of each individual were photographed using a light microscope (Eclipse 80i; Nikon, Tokyo, Japan) with an attached digital camera (Digital Sight DS-Fi1c; Nikon). Then, each nucleus was evaluated using ImageJ version $1.47 \mathrm{t}$ image processing software [16] to determine both the TAA/TNA ratio and the mean AgNOR number via the "freehand selection" tool for each nucleus.

\subsection{Statistical Analysis}

The research data were uploaded and analyzed using the Statistical Package for Social Sciences (IBM Corp., Armonk, NY, USA) version 23.0. The distribution of the data was examined using the Kolmogorov-Smirnov test. The independent samples $t$-test and Mann-Whitney $U$ test were used in comparisons of the variables with and without normal distribution, respectively. The descriptive statistic and Mann-Whitney U tests were used for pairwise comparison of groups. Additionally, a polynomial regression test was performed. Moreover, Bayesian statistics based on the receiver operating characteristic (ROC)-derived cut-off values were calculated. $p<0.05$ was accepted as statistically significant.

\section{Results}

In total, 140 individuals (75 with STEMI and 65 as the control) were included in the current study. The male and female sex frequency was $80 \%$ and $20 \%$ for both groups $(p=1)$. Among the laboratory findings of the groups, the fasting blood sugar, creatinine (mg/dL), WBCs (white blood cells) ( $\mu \mathrm{L} / \mathrm{mL})$, monocytes, neutrophils, and neutrophil/lymphocyte ratio were significantly higher in the STEMI group than in the control group $(p<0.05$ for all) (Table 1). Conversely, LDL (mg/dL), HDL (mg/dL), and hemoglobin (g/dL) were significantly lower in STEMI patients than in the control group ( $p<0.05$ for all) (Table 1$)$. According to the echocardiographic findings, while the interventricular septum thickness (IVST) was significantly higher in the STEMI group $(p<0.001)$, conversely, the ejection fraction (EF) value was significantly lower in the STEMI group $(p<0.001)$. Moreover, both mitral regurgitation (MR) and tricuspid regurgitation (TR) were significantly higher in the STEMI group than in the control ( $p<0.05$ for all) (Table 1$)$.

Moreover, the mean AgNOR number (2.56 \pm 0.8 vs. $1.32 \pm 0.49)$ and TAA/TNA ratio $(0.11 \pm 0.03$ vs. $0.03 \pm 0.01)$ were significantly higher in the STEMI group than in the control $(p<0.001)$. Silver-stained NORs in the lymphocytes of STEMI (a, b, c, and d), and control (e, f, g, and h) group members (×100 magnification) are shown in Figure 1.

When the TAA/TNA ratio was considered, statistically significant relations between IVST and TAA/TNA $(p<0.001)$, fasting blood sugar and TAA/TNA $(p=0.020)$, creatinine and TAA/TNA $(p=0.014)$, HDL $(\mathrm{mg} / \mathrm{dL})$ and TAA/TNA $(p=0.002)$, hemoglobin $(\mathrm{g} / \mathrm{dL})$ and TAA/TNA $(p=0.012)$, WBCs $(\mu \mathrm{L} / \mathrm{mL})$ and TAA/TNA $(p<0.001)$, monocytes $\left(\times 10^{3}\right)$ and TAA/TNA $(p<0.001)$, neutrophils $\left(\times 10^{3}\right)$ and TAA/TNA $(p<0.001)$, neutrophil/lymphocyte ratio and TAA/TNA $(p<0.001)$, and EF and TAA/TNA $(p<0.001)$ were detected (Figure 2 and Table 2).

Additionally, when the mean AgNOR number was considered, statistically significant relations between IVST and the mean AgNOR number $(p=0.002)$, fasting blood sugar and the mean AgNOR number $(p=0.011)$, creatinine and the mean AgNOR number $(p=0.033)$, LDL and the mean AgNOR number $(p=0.005)$, HDL and the mean AgNOR number $(p<0.001)$, hemoglobin $(\mathrm{g} / \mathrm{dL})$ and the mean AgNOR number $(p=0.001), \mathrm{WBC}(\mu \mathrm{L} / \mathrm{mL})$ and the mean AgNOR number $(p<0.001)$, monocytes and the mean AgNOR number $(p=0.010)$, neutrophils and the mean AgNOR number $(p<0.001)$, neutrophil/lymphocyte ratio and the mean AgNOR number $(p<0.001)$, and EF and the mean AgNOR number $(p<0.001)$ were detected (Figure 3 and Table 3$)$. 
Table 1. Demographic, laboratory, and echocardiographic findings of both groups.

\begin{tabular}{|c|c|c|c|c|}
\hline & $\begin{array}{l}\text { STEMI }(n=75) \text { Mean } \pm \text { SD } \\
(\text { Min-Max) (Median) }\end{array}$ & $\begin{array}{l}\text { Control }(n=65) \text { Mean } \pm \text { SD } \\
(\text { Min-Max) (Median) }\end{array}$ & $\chi^{2}$ & $p$ \\
\hline $\operatorname{Sex}(M / F)(\%)$ & $60(80 \%) / 15(20 \%)$ & $52(80 \%) / 13(20 \%)$ & 0.000 & 1.000 \\
\hline Diabetes mellitus (Yes/No) (\%) & $36(48 \%) / 39(52 \%)$ & $21(32.3 \%) / 44(67.7 \%)$ & 3.552 & 0.059 \\
\hline Hypertension (Yes/No) (\%) & $40(53.3 \%) / 35(46.7 \%)$ & $37(56.9 \%) / 28(43.1 \%)$ & 0.81 & 0.670 \\
\hline Hyperlipidemia (Yes/No) (\%) & $38(50.7 \%) / 37(49.3 \%)$ & $33(50.8 \%) / 32(49.2 \%)$ & 0.000 & 0.990 \\
\hline FHCD (Yes/No) (\%) & $38(50.7 \%) / 37(49.3 \%)$ & $27(41.5 \%) / 38(58.5 \%)$ & 1.168 & 0.280 \\
\hline \multirow[t]{2}{*}{ Smoking (Yes/No) (\%) } & $37(49.3 \%) / 38(50.7 \%)$ & $31(47.7 \%) / 34(52.3 \%)$ & 0.038 & 0.846 \\
\hline & & & $\mathbf{Z}$ & $p$ \\
\hline Age (years) & $58.96 \pm 10.37(34-75)(59)$ & $56.29 \pm 10.14(36-75)(57)$ & -1.519 & 0.129 \\
\hline BMI at diagnosis $\left(\mathrm{kg} / \mathrm{m}^{2}\right)$ & $27.86 \pm 1.74(21.78-32.08)(27.78)$ & $27.62 \pm 3.16(20.96-34.72)(27.31)$ & -1.216 & 0.224 \\
\hline Systolic blood pressure (mmHg) & $135.33 \pm 9.74(100-160)(140)$ & $134.46 \pm 12.09(110-160)(140)$ & -0.362 & 0.717 \\
\hline Diastolic blood pressure (mmHg) & $86.47 \pm 6.03(60-100)(90)$ & $85.62 \pm 7.04(70-100)(85)$ & -1.065 & 0.224 \\
\hline Fasting blood sugar (mg/dL) & $117.81 \pm 30.49(76-175)(104)$ & $106.58 \pm 27.44(70-170)(95)$ & -1.996 & 0.046 \\
\hline Creatinine $(\mathrm{mg} / \mathrm{dL})$ & $0.94 \pm 0.21(0.48-1.40)(0.91)$ & $0.85 \pm 0.15(0.5-1.2)(0.86)$ & -2.272 & 0.023 \\
\hline $\mathrm{LDL}(\mathrm{mg} / \mathrm{dL})$ & $112.55 \pm 38.48(28-211)(109)$ & $118.32 \pm 36.27(49-212)(118)$ & -0.986 & 0.324 \\
\hline $\mathrm{HDL}(\mathrm{mg} / \mathrm{dL})$ & $38.77 \pm 9.13(21-59)(38)$ & $44.63 \pm 10.25(23-70)(45)$ & -3.474 & 0.001 \\
\hline Triglyceride (mg/dL) & $186.81 \pm 148.73(48-900)(144)$ & $159.54 \pm 94.58(47-594)(135)$ & -0.345 & 0.730 \\
\hline Total cholesterol (mg/dL) & $186.25 \pm 50.14(93-359)(181)$ & $196.55 \pm 44.17(120-320)(193)$ & -1.617 & 0.106 \\
\hline Hemoglobin (g/dL) & $13.54 \pm 1.54(10-16.9)$ & $14.20 \pm 1.53(10-16.8)$ & -2.663 & 0.008 \\
\hline $\mathrm{WBC}(\mu \mathrm{L} / \mathrm{mL})$ & $\begin{array}{l}11,410.67 \pm 2942.68 \\
(5700-19,200)(12,000)\end{array}$ & $\begin{array}{l}7013.85 \pm 1652.16 \\
(4100-10,900)(6800)\end{array}$ & -8.103 & $<0.001$ \\
\hline Platelet $\left(\times 10^{3}\right)$ & $249.39 \pm 57.65(137-432)(255)$ & $261.78 \pm 61.98(142-467)(253)$ & -0.913 & 0.361 \\
\hline Lymphocyte $\left(\times 10^{3}\right)$ & $2.35 \pm 1.48(0.37-8)(2)$ & $2.18 \pm 0.61(0.79-4.15)(2.18)$ & -0.493 & 0.622 \\
\hline Monocyte $\left(\times 10^{3}\right)$ & $0.80 \pm 0.44(0.1-2.6)(0.75)$ & $0.53 \pm 0.16(0.24-1.02)(0.53)$ & -4.607 & $<0.001$ \\
\hline Neutrophil $\left(\times 10^{3}\right)$ & $8.19 \pm 2.77(3.41-15.50)(8.2)$ & $4.06 \pm 1.17(1.29-6.97)(4.1)$ & -8.586 & $<0.001$ \\
\hline Neutrophil/lymphocyte & $5.33 \pm 5.26(0.84-31.57)(3.68)$ & $1.99 \pm 0.88(0.57-6.97)(1.89)$ & -6.211 & $<0.001$ \\
\hline Mean AgNOR number & $2.56 \pm 0.8(1.23-5)(2.5)$ & $1.32 \pm 0.49(1-3)(1)$ & -8.716 & $<0.001$ \\
\hline \multirow[t]{3}{*}{ TAA/TNA } & $0.11 \pm 0.03(0.04-0.18)(0.11)$ & $0.03 \pm 0.01(0.02-0.06)(0.03)$ & -10.104 & $<0.001$ \\
\hline & Echocardiographic Findings & & & \\
\hline & STEMI Mean \pm SD (Min-Max) & $\begin{array}{l}\text { Control Mean } \pm \text { SD } \\
\text { (Min-Max) }\end{array}$ & $\mathbf{Z}$ & $p$ \\
\hline IVST $(\mathrm{cm})$ & $1.16 \pm 0.12(0.9-1.5)(1.2)$ & $1.08 \pm 0.13(0.9-1.5)(1)$ & -4.012 & $<0.001$ \\
\hline \multirow[t]{2}{*}{$\mathrm{EF}(n \%)$} & $47.69 \pm 7.89(20-60)(50)$ & $63.62 \pm 2.91(50-65)(65)$ & -10.051 & $<0.001$ \\
\hline & STEMI $n(\%)$ & Control $n(\%)$ & $\chi^{2}$ & $p$ \\
\hline MR (Yes/No) (\%) & $52(69.3 \%) / 23(30.7 \%)$ & $21(32.3 \%) / 44(67.7 \%)$ & 19.130 & $<0.001$ \\
\hline AR (Yes/No) (\%) & $12(17.3 \%) / 62(82.7 \%)$ & $7(10.58 \%) / 58(89.2 \%)$ & 1.225 & 0.268 \\
\hline PR (Yes/No) (\%) & $12(16 \%) / 63(84 \%)$ & $5(7.7 \%) / 60(92.3 \%)$ & 2.253 & 0.133 \\
\hline TR (Yes /No) (\%) & $45(60 \%) / 30(40 \%)$ & $24(36.9 \%) / 41(63.1 \%)$ & 7.419 & 0.006 \\
\hline
\end{tabular}

BMI: Body mass index; FHCD: family history of cardiovascular disease; HDL: high-density lipoprotein LDL: low-density lipoprotein; WBC: white blood cells; MR: mitral regurgitation; AR: aortic regurgitation; TR: tricuspid regurgitation; PR: pulmonary regurgitation; IVST: interventricular septum thickness; Min-Max: minimum-maximum; SD: standard deviation; TAA: total AgNOR area; TNA: total nuclear area; AgNOR: argyrophilic nucleolar-organizing region; EF: ejection fraction. The cardiovascular risk factors are shown in Table 2. 
Table 2. Model summary and parameter estimates for TAA/TNA and IVST, fasting blood sugar, creatinine, HDL, hemoglobin, WBC, monocyte, neutrophil, and neutrophil/lymphocyte ratio of both groups.

\begin{tabular}{|c|c|c|c|c|c|c|c|c|c|c|}
\hline \multirow[b]{2}{*}{ Variable } & \multirow[b]{2}{*}{ Equation } & \multicolumn{5}{|c|}{ Model Summary } & \multicolumn{4}{|c|}{ Parameter Estimates } \\
\hline & & $\mathbf{R}^{2}$ & F & df1 & df2 & sig & Constant & b1 & b2 & b3 \\
\hline \multirow{3}{*}{$\begin{array}{l}\text { IVST and } \\
\text { TAA/TNA }\end{array}$} & Linear & 0.102 & 15.615 & 1 & 138 & $<0.001$ & 1.049 & 0.943 & & \\
\hline & $\log$ & 0.090 & 13.574 & 1 & 138 & $<0001$ & 1.289 & 0.061 & & \\
\hline & Cubic & 0.113 & 5.759 & 3 & 136 & 0.001 & 1.178 & -4.961 & 6.959 & -233.473 \\
\hline \multirow{3}{*}{$\begin{array}{l}\text { Fasting blood } \\
\text { sugar and } \\
\text { TAA/TNA } \\
\end{array}$} & Linear & 0.039 & 5.564 & 1 & 138 & 0.020 & 102.487 & 132.121 & & \\
\hline & $\log$ & 0.038 & 5.511 & 1 & 138 & 0.020 & 137.498 & 9.020 & & \\
\hline & Cubic & 0.040 & 1.892 & 3 & 136 & 0.134 & 93.230 & 536.768 & -4423.553 & $13,886.456$ \\
\hline \multirow{3}{*}{$\begin{array}{l}\text { Creatinine and } \\
\text { TAA/TNA }\end{array}$} & Linear & 0.043 & 6.237 & 1 & 138 & 0.014 & 0.828 & 0.894 & & \\
\hline & Log & 0.039 & 5.627 & 1 & 138 & 0.019 & 1.057 & 0.058 & & \\
\hline & Cubic & 0.070 & 3.387 & 3 & 136 & 0.020 & 1.071 & -10.944 & 148.978 & -542.843 \\
\hline \multirow{3}{*}{$\begin{array}{l}\mathrm{HDL}(\mathrm{mg} / \mathrm{dL}) \\
\text { and TAA/TNA }\end{array}$} & Linear & 0.061 & 9.020 & 1 & 138 & 0.003 & 45.829 & -56.646 & & \\
\hline & $\log$ & 0.069 & 10.169 & 1 & 138 & 0.002 & 30.151 & -4.109 & & \\
\hline & Cubic & 0.088 & 4.379 & 3 & 136 & 0.006 & 42.925 & 145.671 & -3452.466 & $15,606.463$ \\
\hline \multirow{3}{*}{$\begin{array}{l}\text { Hemoglobin } \\
\text { (g/dL) and } \\
\text { TAA/TNA }\end{array}$} & Linear & 0.036 & 5.204 & 1 & 138 & 0.024 & 14.368 & -6.789 & & \\
\hline & Log & 0.045 & 6.427 & 1 & 138 & 0.012 & 12.426 & -0.515 & & \\
\hline & Cubic & 0.052 & 2.462 & 3 & 136 & 0.065 & 14.738 & -15.514 & -26.853 & 554.055 \\
\hline \multirow{3}{*}{$\begin{array}{l}\text { WBC }(\mu \mathrm{L} / \mathrm{mL}) \\
\text { and TAA/TNA }\end{array}$} & Linear & 0.358 & 76.924 & 1 & 138 & $<0.001$ & 5965.689 & $44,466.344$ & & \\
\hline & $\log$ & 0.373 & 81.993 & 1 & 138 & $<0.001$ & $17,959.856$ & 3112.044 & & \\
\hline & Cubic & 0.406 & 31.038 & 3 & 136 & $<0.001$ & 7449.484 & $-53,605.833$ & $1,618,705.263$ & $-7,182,084.69$ \\
\hline \multirow{3}{*}{$\begin{array}{c}\text { Monocyte }\left(\times 10^{3}\right) \\
\text { and TAA/TNA }\end{array}$} & Linear & 0.096 & 14.727 & 1 & 138 & $<0.001$ & 0.478 & 2.560 & & \\
\hline & Log & $\begin{array}{l}0.090 \\
0.099\end{array}$ & 15.123 & $\begin{array}{l}1 \\
1\end{array}$ & $\begin{array}{l}130 \\
138\end{array}$ & $<0.001$ & $\begin{array}{l}0.4 / 0 \\
1.164\end{array}$ & 0.178 & & \\
\hline & Cubic & 0.111 & 5.670 & 3 & 136 & 0.001 & 0.652 & -7.112 & 139.691 & -569.051 \\
\hline \multirow{3}{*}{$\begin{array}{l}\text { Neutrophil } \\
\left(\times 10^{3}\right) \text { and } \\
\text { TAA/TNA }\end{array}$} & Linear & 0.344 & 72.240 & 1 & 138 & $<0.001$ & 3.210 & 40.017 & & \\
\hline & $\log$ & 0.372 & 81.584 & 1 & 138 & $<0.001$ & 14.151 & 2.854 & & \\
\hline & Cubic & 0.425 & 33.512 & 3 & 136 & $<0.001$ & 4.238 & -43.604 & 1550.651 & -7314.028 \\
\hline \multirow{3}{*}{$\begin{array}{c}\text { Neutrophil/ } \\
\text { lymphocyte and } \\
\text { TAA/TNA }\end{array}$} & Linear & 0.130 & 20.698 & 1 & 138 & $<0.001$ & 1.128 & 34.698 & & \\
\hline & $\log$ & 0.139 & 22.363 & 1 & 138 & $<0.001$ & 10.577 & 2.461 & & \\
\hline & Cubic & 0.172 & 9.410 & 3 & 136 & $<0.001$ & 3.799 & -122.341 & 2379.721 & $-10,018.401$ \\
\hline \multirow{3}{*}{ EF and TAA/TNA } & Linear & 0.509 & 142.807 & 1 & 138 & 0.000 & 67.518 & -162.426 & & \\
\hline & $\log$ & 0.549 & 167.926 & 1 & 138 & 0.000 & 23.139 & -11.573 & & \\
\hline & Cubic & 0.572 & 60.493 & 3 & 136 & 0.000 & 75.085 & -405.136 & 1204.423 & 1787.206 \\
\hline
\end{tabular}

IVST: Interventricular septum thickness; EF: ejection fraction; WBC: white blood cell; HDL: high-density lipoprotein; TAA: total AgNOR area; TNA: total nuclear area; AgNOR: argyrophilic nucleolar-organizing region.
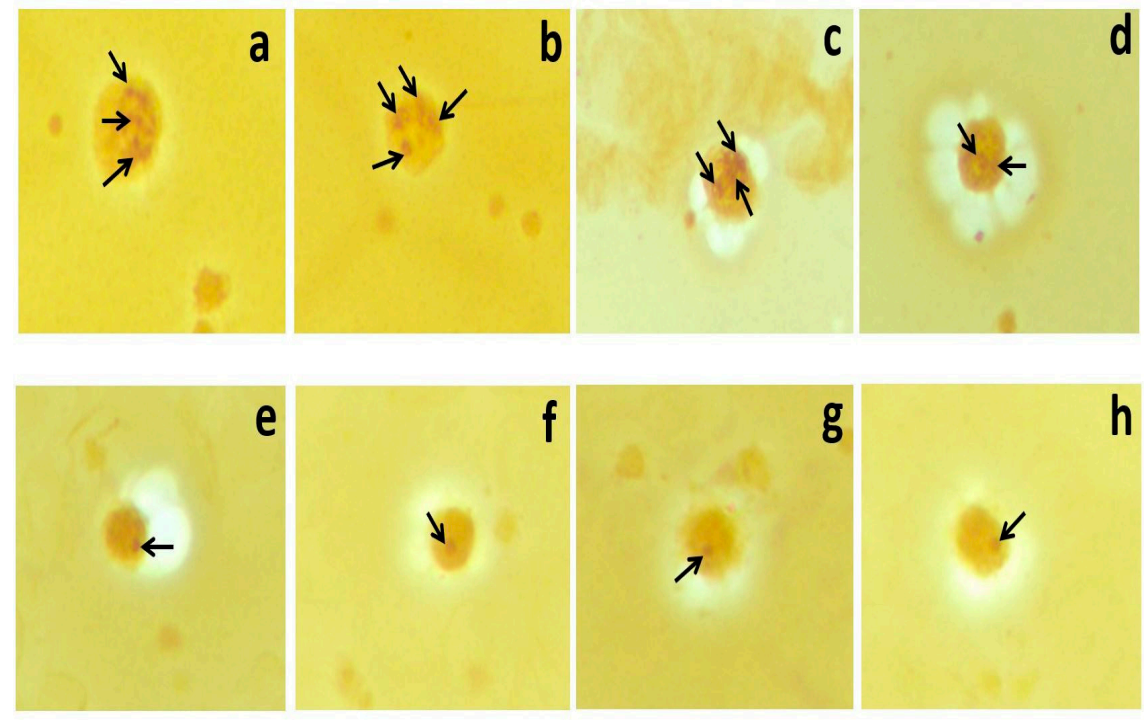

Figure 1. Silver-stained NORs in the lymphocytes of STEMI (a-d) and control (e-h) group members (×100 magnification). 

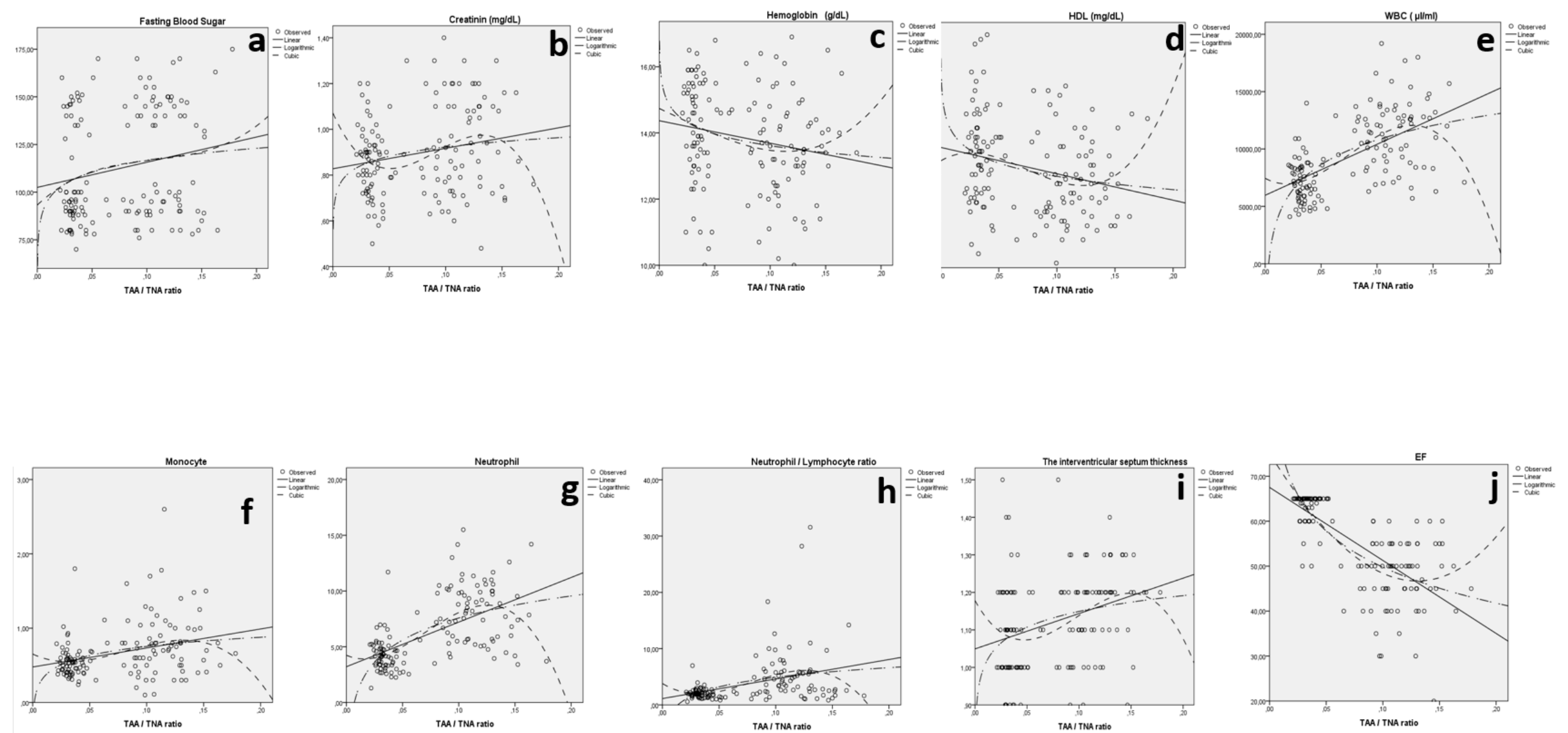

Figure 2. The relation between fasting blood sugar and TAA/TNA ratio (a), creatinine and TAA/TNA ratio $(\mathbf{b})$, hemoglobin and TAA/TNA ratio (c), HDL and TAA/TNA ratio (d), WBCs and TAA/TNA ratio (e), monocytes and TAA/TNA ratio (f), neutrophils and TAA/TNA ratio (g), neutrophil/lymphocyte ratio and TAA/TNA ratio (h), IVST and TAA/TNA ratio (i), and EF and TAA/TNA ratio (j) for both groups. 

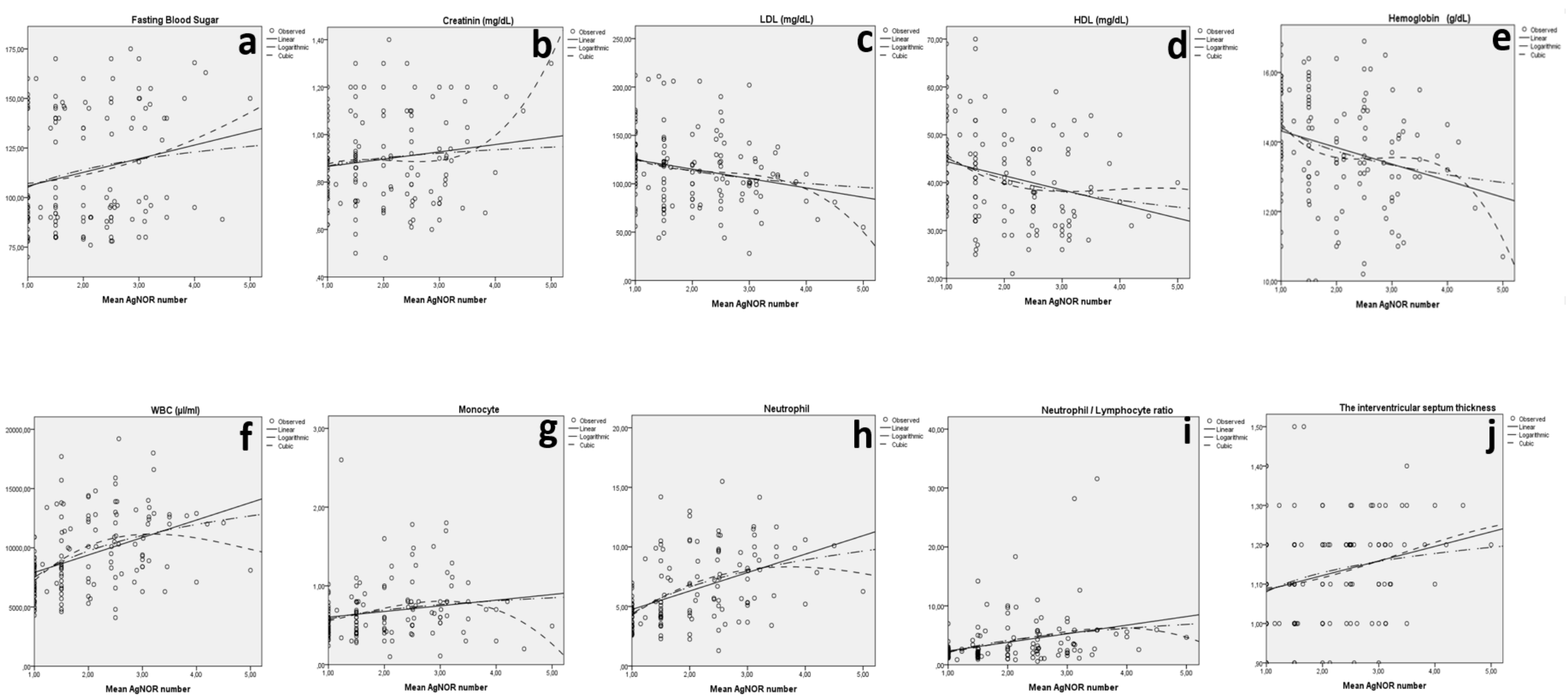

Figure 3. The relation between fasting blood sugar and the mean AgNOR number (a), creatinine and the mean AgNOR number (b), LDL and the mean AgNOR number (c), HDL and the mean AgNOR number (d), hemoglobin and the mean AgNOR number (e), WBCs and the mean AgNOR number (f), monocytes and the mean AgNOR number (g), neutrophils and the mean AgNOR number (h), neutrophil/lymphocyte ratio and the mean AgNOR number (i), and IVST and the mean AgNOR number (j) for both groups. 
Table 3. Model summary and parameter estimates for the mean AgNOR number and IVST, fasting blood sugar, creatinine, LDL, HDL, hemoglobin, WBCs, monocytes, neutrophils, and neutrophil/lymphocyte ratio of both groups.

\begin{tabular}{|c|c|c|c|c|c|c|c|c|c|c|}
\hline \multirow[b]{2}{*}{ Variable } & \multirow[b]{2}{*}{ Equation } & \multicolumn{5}{|c|}{ Model Summary } & \multicolumn{4}{|c|}{ Parameter Estimates } \\
\hline & & $\mathbf{R}^{2}$ & F & df1 & df2 & sig & Constant & b1 & b2 & b3 \\
\hline \multirow{3}{*}{$\begin{array}{l}\text { IVST and } \\
\text { mean AgNOR }\end{array}$} & Linear & 0.068 & 10.014 & 1 & 138 & 0.002 & 1.048 & 0.037 & & \\
\hline & $\log$ & 0.061 & 8.983 & 1 & 138 & 0.003 & 1.081 & 0.070 & & \\
\hline & Cubic & 0.069 & 3.360 & 3 & 136 & 0.021 & 1.093 & -0.021 & 0.021 & -0.002 \\
\hline \multirow{3}{*}{$\begin{array}{l}\text { Fasting blood sugar } \\
\text { and mean AgNOR }\end{array}$} & Linear & 0.045 & 6.565 & 1 & 138 & 0.011 & 98.958 & 6.875 & & \\
\hline & $\log$ & 0.040 & 5.719 & 1 & 138 & 0.018 & 105.125 & 12.857 & & \\
\hline & Cubic & 0.048 & 2.290 & 3 & 136 & 0.081 & 106.272 & -0.889 & 1.763 & -0.024 \\
\hline \multirow{3}{*}{$\begin{array}{l}\text { Creatinine and } \\
\text { mean AgNOR }\end{array}$} & Linear & 0.022 & 3.113 & 1 & 138 & 0.080 & 0.835 & 0.031 & & \\
\hline & $\log$ & 0.014 & 2.022 & 1 & 138 & 0.157 & 0.867 & 0.050 & & \\
\hline & Cubic & 0.062 & 2.997 & 3 & 136 & 0.033 & 0.718 & 0.269 & -0.132 & 0.021 \\
\hline \multirow{3}{*}{$\begin{array}{l}\text { LDL (mg/dL) and } \\
\text { mean AgNOR }\end{array}$} & Linear & 0.056 & 8.205 & 1 & 138 & 0.005 & 134.453 & -9.686 & & \\
\hline & $\log$ & 0.051 & 7.408 & 1 & 138 & 0.007 & 125.951 & -18.438 & & \\
\hline & Cubic & 0.068 & 3.283 & 3 & 136 & 0.023 & 162.666 & -56.427 & 22.083 & -3.059 \\
\hline \multirow{3}{*}{$\begin{array}{l}\mathrm{HDL}(\mathrm{mg} / \mathrm{dL}) \text { and } \\
\text { mean AgNOR }\end{array}$} & Linear & 0.072 & 10.783 & 1 & 138 & 0.001 & 47.366 & -2.960 & & \\
\hline & $\log$ & 0.086 & 12.950 & 1 & 138 & $<0.001$ & 45.233 & -6.433 & & \\
\hline & Cubic & 0.094 & 4.702 & 3 & 136 & 0.004 & 58.798 & -17.121 & 4.00 & -0.396 \\
\hline \multirow{3}{*}{$\begin{array}{l}\text { Hemoglobin }(\mathrm{g} / \mathrm{dL}) \\
\text { and mean AgNOR }\end{array}$} & Linear & 0.078 & 11.638 & 1 & 138 & 0.001 & 14.796 & -0.477 & & \\
\hline & $\log$ & 0.083 & 12.499 & 1 & 138 & 0.001 & 14.422 & -0.986 & & \\
\hline & Cubic & 0.103 & 5.201 & 3 & 136 & 0.002 & 17.819 & -4.878 & 1.14 & -0.221 \\
\hline \multirow{3}{*}{$\begin{array}{l}\mathrm{WBC}(\mu \mathrm{L} / \mathrm{mL}) \text { and } \\
\text { mean AgNOR }\end{array}$} & Linear & 0.170 & 28.298 & 1 & 138 & $<0.001$ & 6444.631 & 1473.871 & & \\
\hline & $\log$ & 0.205 & 35.527 & 1 & 138 & $<0.001$ & 7491.555 & 3229.917 & & \\
\hline & Cubic & 0.222 & 12.970 & 3 & 136 & $<0.001$ & 1688.337 & 7049.536 & -1626.10 & 108.735 \\
\hline \multirow{3}{*}{$\begin{array}{c}\text { Monocyte }\left(\times 10^{3}\right) \text { and } \\
\text { mean AgNOR }\end{array}$} & Linear & 0.033 & 4.706 & 1 & 138 & 0.032 & 0.531 & 0.072 & & \\
\hline & $\log$ & 0.046 & 6.694 & 1 & 138 & 0.011 & 0.575 & 0.170 & & \\
\hline & Cubic & 0.079 & 3.915 & 3 & 136 & 0.010 & 0.452 & 0.035 & 0.091 & -0.021 \\
\hline \multirow{3}{*}{$\begin{array}{l}\text { Neutrophil }\left(\times 10^{3}\right) \text { and } \\
\text { mean AgNOR }\end{array}$} & Linear & 0.223 & 39.523 & 1 & 138 & $<0.001$ & 3.201 & 1.548 & & \\
\hline & $\log$ & 0.250 & 46.118 & 1 & 138 & $<0.001$ & 4.366 & 3.281 & & \\
\hline & Cubic & 0.256 & 15.635 & 3 & 136 & $<0.001$ & 0.078 & 5.073 & -0.944 & 0.047 \\
\hline \multirow{3}{*}{$\begin{array}{c}\text { Neutrophil/lymphocyte } \\
\text { ratio and } \\
\text { mean AgNOR }\end{array}$} & Linear & 0.099 & 15.237 & 1 & 138 & $<0.001$ & 0.894 & 1.456 & & \\
\hline & $\log$ & 0.104 & 16.073 & 1 & 138 & $<0.001$ & 2.051 & 2.980 & & \\
\hline & Cubic & 0.108 & 5.515 & 3 & 136 & 0.001 & 1.545 & -0.244 & 1.076 & -0.181 \\
\hline \multirow{3}{*}{ EF and mean AgNOR } & Linear & 0.260 & 48.550 & 1 & 138 & $<0.001$ & 66.169 & -5.585 & & \\
\hline & $\log$ & 0.315 & 63.584 & 1 & 138 & $<0.001$ & 62.228 & -1.285 & & \\
\hline & Cubic & 0.351 & 24.483 & 3 & 136 & $<0.001$ & 92.315 & -38.908 & 11.372 & -1.070 \\
\hline
\end{tabular}

IVST: Interventricular septum thickness; WBC: white blood cell; HDL: high-density lipoprotein; LDL: low-density lipoprotein; AgNOR: argyrophilic nucleolar-organizing region; EF: ejection fraction.

The troponin values of patients were $40.4 \pm 22.3$. Additionally, when both the mean AgNOR number and the TAA/TNA ratio were considered, there were no statistically significant relations between the levels of troponin and both AgNOR amounts (mean AgNOR number and TAA/TNA) $(p>0.05)$. The Bayesian statistics based on the receiver operating characteristic (ROC)-derived cut-off values are shown in Table 4.

Table 4. Bayesian statistics based on ROC-derived cut-off values.

\begin{tabular}{|c|c|c|c|c|c|c|}
\hline & Groups & $\operatorname{AUC}(95 \%)$ & Cut-Off & $p$ & Sensitivity (\%) & Specificity (\%) \\
\hline Mean AgNOR number & \multirow{2}{*}{$\begin{array}{l}\text { STEMI: } 75 \text { and } \\
\text { Control: } 65\end{array}$} & $\begin{array}{c}0.923 \\
(0.879-0.967)\end{array}$ & 1.523 & 0.000 & 86.7 & 86.2 \\
\hline TAA/TNA & & $0.996(0.988-1)$ & 0.054 & 0.000 & 98.7 & 98.5 \\
\hline
\end{tabular}

AUC: Area under the ROC curve; TAA: total AgNOR area. 


\section{Discussion}

The present study showed that both the TAA/TNA ratio and the mean AgNOR number were significantly higher in the STEMI patients compared to the subjects without acute coronary syndrome.

In our previous studies, we detected that the AgNOR proteins increased depending on the hypoxic condition caused by $\mathrm{CO}$ exposure [9]. At the same time, it was reported that the TAA/TNA ratio could be used instead of histopathological evaluation scores as a biomarker for obtaining knowledge about the myocardial damage degree in rats [9]. Moreover, we reported that the TAA/TNA ratio could be used as an indicator for detection of the CO exposure level that causes hypoxia [17]. Additionally, we learned that knowledge about cardiomyopathy $(\mathrm{CM})$ levels may be obtained via AgNOR proteins, and these proteins may be used instead of carboxyhemoglobin $(\mathrm{COHb})$ to detect $\mathrm{CO}$ intoxication levels [10]. In acute coronary thrombosis, myocardial ischemia/infarction, and the reperfusion process, it is highly probable that the molecular protective mechanisms of immune system cells will be activated. In our study, because the mean AgNOR number and TAA/TNA ratios were significantly higher in STEMI patients compared to the control group, it may be considered a protective reaction of the immune system in the early period of MI.

When the TAA/TNA ratio was taken into consideration, statistically significant relations between EF and TAA/TNA, IVST and TAA/TNA, fasting blood sugar and TAA/TNA, creatinine and TAA/TNA, HDL and TAA/TNA, and hemoglobin (g/dL) and TAA/TNA were detected. Additionally, statistically significant relations between EF and the mean AgNOR number, IVST and the mean AgNOR number, fasting blood sugar and the mean AgNOR number, creatinine and the mean AgNOR number, LDL $(\mathrm{mg} / \mathrm{dL})$ and the mean AgNOR number, HDL (mg/dL) and the mean AgNOR number, and hemoglobin (g/dL) and the mean AgNOR number were detected. Considering the relationship between interventricular septum thickness and hypertension, the statistically significant relationship between the TAA/TNA ratio, the mean AgNOR number, and cardiovascular risk factors such as hypertension, dyslipidemia, high blood sugar, creatinine, and anemia suggests that AgNOR proteins may have some important roles that we have not yet identified in the pathophysiology of atherosclerotic cardiovascular disease.

In addition, we found that both the mean AgNOR number and the TAA/TNA ratio increased as the EF decreased. Based on this, it can be said that AgNOR parameters increase as the severity of myocardial infarction increases or the damage to myocardial tissue increases. According to the current study, the mean AgNOR number allowed 86.7\% sensitivity and $86.2 \%$ specificity and the TAA/TNA ratio allowed $98.7 \%$ sensitivity and 98.5\% specificity for the discrimination of the control group from the STEMI group (Table 4).

According to the literature, high WBC levels are considered an independent predictor of acute myocardial infarction because of their association with more complications, more extensive myocardial necrosis, and worse outcomes [18-21]. In our study, in line with the literature, WBC count, neutrophil count, neutrophil/lymphocyte ratio, and monocyte count were significantly higher in STEMI patients compared to the control group.

In addition, from the data of our study, we found a statistically significant relationship between both AgNOR parameters (TAA/TNA and the mean AgNOR number) and WBCs, neutrophils, the neutrophil/lymphocyte ratio, and monocytes. The correlation between AgNOR proteins and parameters such as WBC count, neutrophil count, neutrophil/lymphocyte ratio, and monocyte count, which are predictors of cardiovascular mortality and morbidity in patients with STEMI, may open the door to a new marker in STEMI. As is known in the diagnosis of STEMI, the fastest diagnostic tool is undoubtedly ECG. In addition, other parameters such as troponin, CRP, and WBC can provide useful information about the prognosis of the disease. Both AgNOR protein amounts increase depending on the hypoxia that occurs in STEMI. Considering that AgNOR proteins are a secondary response to inflammation, they are likely to provide some prognostic information. In addition, if this work is supported by larger studies in the future, it may 
benefit diagnosis and prognosis in the non-ST-elevation myocardial infarction (NSTEMI) patient group.

The limitations of present study are the relatively small study population and the single-center nature of the study.

\section{Conclusions}

In conclusion, we suggest that AgNOR parameters could be a reliable marker in patients with STEMI. Prospective studies with a larger cohort are required to establish its prognostic role in this population.

Author Contributions: Conceptualization, I.H.D. and R.E.; formal analysis, İ.H.D. and R.E.; investigation, İ.H.D. and R.E.; methodology, İ.H.D. and R.E.; visualization, İ.H.D. and R.E.; writing一original draft, İ.H.D. and R.E.; writing-review and editing, İ.H.D. and R.E. All authors have read and agreed to the published version of the manuscript.

Funding: This research received no external funding.

Institutional Review Board Statement: The study was conducted according to the guidelines of the Declaration of Helsinki and approved by the local Ethics Committee of Duzce University (ethical approval code: 2021/62).

Informed Consent Statement: Informed consent was obtained from all subjects involved in the study.

Data Availability Statement: The data presented in this study are available on request from the corresponding author. The data are not publicly available since the people participating in the study have not allowed their data to be shared with third parties.

Acknowledgments: We thank all the participants involved in the study.

Conflicts of Interest: The authors declare that no conflict of interest.

\section{References}

1. Damar, İ.H.; Eroz, R. The Association of Hereditary Prothrombotic Risk Factors with ST-Elevation Myocardial Infarction. Medeni. Med. J. 2020, 35, 295-303. [CrossRef] [PubMed]

2. Zheng, Y.; Wang, Z.; Deng, L.; Yuan, X.; Ma, Y.; Zhang, G.; Gantier, M.P.; Liu, J.P.; Shen, L.; Xu, D. Osteopontin promotes in flammation in patients with acute coronary syndrome through its activity on IL-17 producing cells. Eur. J. Immunol. 2012, 42, 2803-2814. [CrossRef] [PubMed]

3. Frangogiannis, N.G.; Mendoza, L.H.; Lindsey, M.L.; Ballantyne, C.M.; Michael, L.H.; Smith, C.W.; Entman, M.L. IL-10 is induced in the reperfused myocardium and may modulate the reaction to injury. J. Immunol. 2000, 165, 2798-2808. [CrossRef] [PubMed]

4. Spagnoli, L.G.; Bonanno, E.; Mauriello, A.; Palmieri, G.; Partenzi, A.; Sangiorgi, G.; Crea, F. Multicentric inflammation in epicardial coronary arteries of patients dying of acute myocardial infarction. J. Am. Coll. Cardiol. 2002, 40, 1579-1588. [CrossRef]

5. Moreira, D.M.; da Silva, R.L.; Vieira, J.L.; Fattah, T.; Lueneberg, M.E.; Gottschall, C.A. Role of vascular inflammation in coronary artery disease: Potential of anti-inflammatory drugs in the prevention of atherothrombosis. Inflammation and anti-inflammatory drugs in coronary artery disease. Am. J. Cardiovasc. Drugs 2015, 15, 1-11. [CrossRef] [PubMed]

6. Eroz, R.; Yilmaz, S.; Cucer, N. Argyrophilic nucleolar organizing region associated protein synthesis in hair root cells of humans at different developmental stages and sex. Biotech. Histochem. 2013, 88, 267-271. [CrossRef] [PubMed]

7. Selvi, B.; Demirtas, H.; Eroz, R.; Imamoglu, N. Reduction of the argyrophilic nucleolar organizing region associated protein synthesis with age in buccal epithelial cells of healthy individuals. Aging Clin. Exp. Res. 2015, 27, 201-208. [CrossRef] [PubMed]

8. Çolakoğlu, S.; Saritas, A.; Eroz, R.; Oktay, M.; Yaykasli, K.O.; Akoz, A.; Kaya, E.; Kandis, H. Is one-time carbon monoxide intoxication harmless? Evaluation by argyrophilic nucleolar-organizing regions staining method. Hum. Exp. Toxicol. 2015, 34, 24-31. [CrossRef] [PubMed]

9. Saritas, A.; Gunes, H.; Colakoglu, S.; Eroz, R.; Akoz, A.; Oktay, M.; Buyukkaya, A.; Kandis, H.; Ozkan, A. Are there any effects of chronic carbon monoxide exposure on argyrophilic nucleolar-organizing region-associated protein synthesis in rat myocardium? Hum. Exp. Toxicol. 2016, 35, 921-928. [CrossRef] [PubMed]

10. Kandiş, H.; Afacan, M.A.; Eröz, R.; Colakoglu, S.; Bayramoglu, A.; Oktay, M.; Saritas, A.; Colaki, S.; Kaya, M.; Kara, İ.H. Can argyrophilic nucleolar organizing region-associated protein amount be used for the detection of cardiac damage? Hum. Exp. Toxicol. 2016, 35, 323-331. [CrossRef]

11. Gunes, H.; Saritas, A.; Eroz, R.; Colakoglu, S. Use of argyrophilic nucleolar-organizer region-associated protein synthesis in skeletal muscle cells for prediction of chronic carbon monoxide exposure. Toxin Rev. 2020, 39, 349-354. [CrossRef]

12. Thygesen, K.; Alpert, J.S.; Jaffe, A.S.; Chaitman, B.R.; Bax, J.J.; Morrow, D.A.; White, H.D.; The Executive Group on behalf of the Joint European Society of Cardiology (ESC)/American College of Cardiology (ACC)/American Heart Association (AHA)/World 
Heart Federation (WHF) Task Force for the Universal Definition of Myocardial Infarction. Fourth Universal Definition of Myocardial Infarction. J. Am. Coll. Cardiol. 2018, 72, 2231-2264. [CrossRef] [PubMed]

13. Lang, R.M.; Badano, L.P.; Mor-Avi, V.; Afilalo, J.; Armstrong, A.; Ernande, L.; Flachskampf, F.A.; Foster, E.; Goldstein, S.A.; Kuznetsova, T.; et al. Recommendations for cardiac chamber quantification by echocardiography in adults: An update from the American Society of Echocardiography and the European Association of Cardiovascular Imaging. J. Am. Soc. Echocardiogr. 2015, 28, 1-39.e14. [CrossRef] [PubMed]

14. Benn, P.A.; Perle, M. Chromosome staining and banding techniques. In Human Cytogenetics, Constitutional Analysis, Practical Approach; Rooney, D.E., Czepulkowski, B.H., Eds.; Oxford University Press: Oxford, UK, 1986; Volume 1, pp. 91-118.

15. Lindner, L.E. Improvements in the silver-staining technique for nucleolar organizer regions (AgNOR). J. Histochem. Cytochem. 1993, 41, 439-445. [CrossRef] [PubMed]

16. Rasband, W.S. ImageJ; U.S. National Institutes of Health: Bethesda, MD, USA, 1997-2016. Available online: https://imagej.nih gov/ij/ (accessed on 27 October 2021).

17. Eroz, R.; Saritas, A.; Colakoglu, S.; Oktay, M.; Kandiş, H. Evaluation of argyrophilic nucleolar organizing region-associated protein synthesis in femoral muscle cells of rats exposed 3000 ppm carbon monoxide gas. Konuralp Med. J. 2016, 8, 9-13.

18. Caimi, G.; Lo Presti, R.; Canino, B.; Ferrera, E.; Hopps, E. Behaviour of the neutrophil to lymphocyte ratio in young subjects with acute myocardial infarction. Clin. Hemorheol. Microcirc. 2016, 62, 239-247. [CrossRef] [PubMed]

19. Park, J.S.; Seo, K.W.; Choi, B.J.; Choi, S.Y.; Yoon, M.H.; Hwang, G.S.; Tahk, S.J.; Shin, J.H. Importance of prognostic value of neutrophil to lymphocyte ratio in patients with ST-elevation myocardial infarction. Medicine 2018, 97, e13471. [CrossRef] [PubMed]

20. Meeuwsen, J.A.L.; Wesseling, M.; Hoefer, I.E.; de Jager, S.C.A. Prognostic Value of Circulating Inflammatory Cells in Patients with Stable and Acute Coronary Artery Disease. Front. Cardiovasc. Med. 2017, 14, 44. [CrossRef] [PubMed]

21. Wang, Z.; Ren, L.; Liu, N.; Lei, L.; Ye, H.; Peng, J. Association of monocyte count on admission with angiographic no-reflow after primary percutaneous coronary intervention in patients with ST-segment elevation myocardial infarction. Kardiol. Pol. 2016, 74, 1160-1166. [CrossRef] [PubMed] 